\title{
Van Gogh's Irises and Roses: the contribution of chemical analyses and imaging to the assessment of color changes in the red lake pigments
}

\author{
Silvia A. Centeno ${ }^{*^{*}} \mathbb{0}$, Charlotte Hale², Federico Carò ${ }^{2}$, Anna Cesaratto ${ }^{1}$, Nobuko Shibayama ${ }^{1}$, John Delaney ${ }^{3}$, \\ Kathryn Dooley ${ }^{3}$, Geert van der Snickt ${ }^{4}$, Koen Janssens ${ }^{4}$ and Susan Alyson Stein ${ }^{5}$
}

\begin{abstract}
Vincent van Gogh's still lifes Irises and Roses were investigated to shed light onto the degree to which the paintings had changed, both individually and in relation to each other since they were painted, particularly in regard to the fading of the red lakes. Non-invasive techniques, including macroscopic X-ray fluorescence mapping, reflectance imaging spectroscopy, and X-radiography, were combined with microanalytical techniques in a select number of samples. The in-depth microchemical analysis was necessary to overcome the complications that arise when evaluating by non-invasive methods alone the compositions of passages with complex layering and mixing of paints. The results obtained by these two approaches were complemented by color measurements performed on paint cross-sections and on protected edges, and with historical information provided by the artist's own descriptions, early reviews and reproductions, and the data was used to carry out digital color simulations that provided, to a certain extent, a visualization of how the paintings may have originally appeared.
\end{abstract}

Keywords: Vincent van Gogh, Irises, Roses, Eosin red lake pigment, Brazilwood, Fading

\section{Background}

Irises and Roses in the collection of The Metropolitan Museum of Art (The Met), Roses in the collection of the National Gallery of Art, Washington, and Irises at the Van Gogh Museum in Amsterdam were examined in preparation for the exhibition Van Gogh: Irises and Roses, that took place at The Met in 2015, curated by Susan Alyson Stein with a technical examination directed by Charlotte Hale [1]. The exhibition reunited the four still lifes, painted as a series in May 1890, shortly before the artist's departure from the asylum of Saint Paul de Mausole in Saint-Rémy, Provence. One of the central aspects considered in the exhibition was the degree to which the paintings had changed, both individually and in relation

\footnotetext{
*Correspondence: silvia.centeno@metmuseum.org

1 Department of Scientific Research, The Metropolitan Museum of Art, 1000 Fifth Avenue, New York, NY 10028, USA

Full list of author information is available at the end of the article
}

to each other, since Van Gogh painted them, particularly in regard to the fading of the red lakes. Digital color simulations of the four paintings were included in a video presentation on the series, shown in the exhibition and on The Met's website.

The colorants present in lake pigments have relatively high tinting powers and, therefore, are used generally in small amounts in objects of cultural significance, which makes them difficult to detect, especially when sampling is limited. UV-vis fluorescence and visible absorption spectrometry have been applied to identify organic colorants in works of art $[2,3]$, however these techniques give spectra with broad features that are not always useful [2]. In the FTIR analysis of lakes, the presence of bands due to the binding media frequently interferes with the identification of the colorants, and the Raman spectroscopic analysis is often limited by the high fluorescence of most organic colorants. Surface enhanced Raman spectroscopy (SERS) gives good results in the analysis of red lake 
pigments and dyes, both in term of specificity and detection limit $[4,5]$, nevertheless the technique has limitations for resolving mixtures of two or more colorants. High performance liquid chromatography (HPLC) is well established for identifying organic colorants in artworks because it permits not only a qualitative identification of the dyes, but also a quantitative estimation and, therefore, it is highly specific. Major limitations of HPLC are the relatively large sample sizes and the complex sample preparation required $[4,6]$. Laser desorption ionization mass spectrometry (LDI-MS) has been applied to detect dyestuffs in works of art [7], and has given good results in samples from archeological objects but not in paint cross-sections due to the interference from the binding media. The major benefits of LDS-MS are the high resolution and the possibility to detect the dye-mordant complexes as a whole [8]. Pyrolysis-gas chromatography/ mass spectrometry (Py-GC/MS) has also been used to identify synthetic organic pigments in paintings. In some cases, this technique produces the same fragments for more than one pigment, but the overall pyrolysis profile generally allows the identification of the individual pigment; however only the most abundant products are typically detected [9].

The approach to the re-colorization of Irises and Roses was based on information gathered using a broad range of analytical techniques, applied in a non-invasive manner and in a few selected microsamples, that included macroscopic X-ray fluorescence (MA-XRF) mapping, reflectance imaging spectroscopy (RIS), fluorescence imaging, X-radiography, Raman spectroscopy, high performance liquid chromatography (HPLC), surface enhanced Raman spectroscopy (SERS), and scanning electron microscopy-energy dispersive X-ray spectrometry (SEM-EDS). The results were complemented by color measurements performed on paint cross-sections and on protected edges, with historical information provided by the artist's own descriptions, early reviews and reproductions, and with what had been previously published on Van Gogh's materials and techniques [10-13].

The use of red organic lake pigments by Vincent van Gogh during different periods of his life and the impact of their permanence on a large number of his paintings has been widely reviewed and discussed by numerous authors (see, for example [11-16]). The present article will focus on the contribution of chemical analyses and imaging to the assessment of color changes due to fading in the paint passages containing organic red pigments and to the virtual color simulations of Irises and Roses in The Met's collection. For more information on other aspects of the technical examination, please see [1].

\section{Experimental MA-XRF}

The MA-XRF instrument used consists of a measuring head that is moved across the painting surface by means of a motorized $\mathrm{X}-\mathrm{Y}$ stage with a maximum travel range of $80 \times 60 \mathrm{~cm}$. The measuring head includes a $30 \mathrm{~W} \mathrm{Rh}$ target micro-focus X-ray tube and a Silicon Drift Detector (SDD) to collect the fluorescence signal. The X-ray source was operated at $50 \mathrm{kV}$ and $0.5 \mathrm{~mA}$, and the resulting beam was focused by means of a polycapillary optic. A Z motor allowed optimising the spot size to the step size by varying the distance between the painting and the measuring head. The overall paintings were scanned at $250 \mathrm{~ms} /$ pixel with a $1 \mathrm{~mm}$ step size. The spectra were processed by employing a combination of PyMca and Datamuncher software as described in [17] and by using the Bruker M6 Jetstream software.

\section{Reflectance imaging spectroscopy (RIS)}

Irises and Roses were examined using hyperspectral reflectance imaging spectroscopy by acquiring reflectance spectra from 400 to $1680 \mathrm{~nm}$ across the surface of each work. The primary interest was to get accurate reflectance spectra of the red paints and their spatial distributions. These spectra and maps were used to identify the pigments present.

Hyperspectral image cubes were collected with optimized whiskbroom line-scanning imaging spectrometers (Surface Optics Corporation). Details of the setup have been described previously [18]. The first spectrometer utilized an EMCCD detector (ProEM1024, Princeton Instruments) operating from 380 to $1000 \mathrm{~nm}$ with $2.5 \mathrm{~nm}$ sampling, at a light level of 1500 lux for $\sim 5$ min per cube. The image cubes are 1024 by 1024 spatial and 280 spectral. The second spectrometer utilized is an InGaAs array (SUI 640SDV, Sensors Unlimited) operating from 967 to $1680 \mathrm{~nm}$ with $3.4 \mathrm{~nm}$ sampling, 1000 lux, and an integration time of $30 \mathrm{~ms}$ per line. The image cubes have 640 by 640 pixels in the spatial dimensions and 256 along the spectral axis. The image cubes were dark-corrected, flat-fielded, and calibrated to apparent reflectance using diffuse reflectance standards (Labsphere Inc.). The calibrated image cubes were spatially registered using a point-based algorithm [19]. Spectral analysis of the image cubes was done using convex geometry methods [20] and mapping methods employing Match Tuned Filters [21]. Only the findings for The Met's Roses are discussed in this article.

Color coordinates ( $\mathrm{L}^{*} \mathrm{a} \mathrm{a}^{*} \mathrm{~b}^{*}$ values) useful for the color simulations were obtained using a fiber optic reflectance spectroradiometer model FS3 (ASD Inc., Boulder Co.). 


\section{Samples}

Selected samples were removed using a scalpel under high magnification. Some of these samples were analyzed as scrapings and some were mounted as cross-sections in Technovit $^{\circledR}$ resin and polished using Micromesh ${ }^{\circledR}$ cloth followed by ion milling with a Hitachi IM4000 ion milling system.

\section{SEM-EDS}

After ion milling, cross-sections were carbon coated prior to SEM-EDS analysis.

Analyses were performed with a FE-SEM Zeiss Sigma $\mathrm{HD}$, equipped with an Oxford Instrument X-Max ${ }^{\mathrm{N}} 80$ SDD detector. Backscattered electron (BSE) images, energy-dispersive spectrometry (EDS) analysis, and X-ray mapping were carried out with an accelerating voltage of $20 \mathrm{kV}$ in high vacuum.

\section{Raman spectroscopy and SERS}

Raman spectroscopy measurements were done on sample scrapings and on the cross-sections using a Renishaw System 1000 coupled to a Leica DM LM microscope. All the spectra were acquired using a $785 \mathrm{~nm}$ laser excitation focused on the samples using a $50 \times$ objective lens, with integration times between 10 and 120 s. A 1200 lines/ $\mathrm{mm}$ grating and a thermoelectrically cooled CCD detector were used. Powers at the sample were set between 0.5 and $5 \mathrm{~mW}$ using neutral density filters.

For the SERS measurements on the sample removed from the background area in Irises, a silver colloid synthesized by microwave-assisted glucose reduction of silver sulphate in the presence of sodium citrate was used as a substrate [4]. A $0.8 \mu \mathrm{L}$ drop of a $1 \% \mathrm{HNO}_{3}$ solution was placed on the sample in order to induce the extraction of the dye and then $2 \mu \mathrm{L}$ of the silver colloid were added on top of the drop. The aggregation of the nanoparticles is induced by the presence of $\mathrm{HNO}_{3}$, which also acts as a pretreatment agent particularly suitable for xanthene lakes [22].

In the case of the samples removed from the tabletop and one of the stems in Roses, it was necessary to carry out a pre-treatment by placing them on a polyethylene sample holder in a micro-chamber filled with HF vapors for $5 \mathrm{~min}$. This step is often fundamental to hydrolyze natural dyes-based lakes and make the free dyes available for adsorption on the nanoparticles, as previously demonstrated [23]. Once the sample holder was removed from the chamber, $2 \mu \mathrm{L}$ of silver colloid were placed directly on the sample followed by $0.5 \mu \mathrm{L}$ of a $0.5 \mathrm{M}$ $\mathrm{KNO}_{3}$ solution to induce the aggregation.

All the SERS spectra were obtained using a Bruker Senterra Raman spectrometer equipped with a chargecoupled device (CCD) detector and a 1800 rulings $/ \mathrm{mm}$ holographic grating providing a resolution of $3-5 \mathrm{~cm}^{-1}$. The $488 \mathrm{~nm}$ radiation emitted by a Spectra Physics Cyan solid state laser was employed as the excitation source, with a power at the sample of about $0.7 \mathrm{~mW}$. The spectra were acquired with two integrations of $15 \mathrm{~s}$ each, focusing just below the top surface of the dye-colloid drop with an Olympus $20 \times$ LMPlanFL long working distance microscope objective.

\section{HPLC}

Methanol and acetonitrile (HPLC grade), hydrofluoric acid (HF), formic acid (88\%), oxalic acid, and pyridine (analytical grade) were purchased from Fisher Scientific (Pittsburgh, PA), and the eosin Y disodium salt was purchased from Sigma-Aldrich (St. Louis, MO).

Deionized water $(18 \mathrm{M} \Omega / \mathrm{cm})$ was produced by a system equipped with mixed bed ion exchange tanks and one micron filters (Evoqua water technologies, NJ), and was subsequently filtered with a $0.45 \mu \mathrm{m}$ Durapore ${ }^{\circledR}$ membrane filter (Fisher Scientific, Pittsburg, PA).

The reference woolen yarn dyed with brazilwood (Caesalpinia echinata Lam.) used in this study was made by conservators in the Textile Conservation Department, The Metropolitan Museum of Art, NY.

The samples (approximately $0.5 \mathrm{~mm} \times 0.5 \mathrm{~mm}$ ) removed from the paintings were ultrasonicated with $20 \mu \mathrm{L}$ of a mixture of acetonitrile and methanol $(1: 1 \mathrm{v} / \mathrm{v})$ for $30 \mathrm{~min}$, and left overnight at RT. $20 \mu \mathrm{L}$ of $4 \mathrm{M}$ aqueous HF were added to the vial, vortexed left at RT for several hours [24] and occasionally ultrasonicated. The HF solution was then evaporated under a nitrogen stream. $40 \mu \mathrm{L}$ of methanol were added to the vial and they were then evaporated under a nitrogen stream. This step was repeated three more times in order to remove HF residues. The extract was dissolved in $7 \mu \mathrm{L}$ of methanol and then $7 \mu \mathrm{L}$ of $0.88 \%$ aqueous formic acid (v/v) were added, subsequently centrifuged at $3500 \mathrm{~g}$ for $10 \mathrm{~min}$ and the supernatant was injected to the HPLC system.

The analytical system used consisted of a $1525 \mu$ binary HPLC pump, 2996 PDA detector, 1500 series column heater, in-line degasser and a Rheodyne 7725i manual injector with $20 \mu \mathrm{L}$ loop (Waters Corporation, Milford MA). A Rheodyne column switching valve (Waters Corporation, Milford MA) was connected between the guard column and the analytical column for the purpose to remove remaining the HF residue in the sample. An Xterra $\mathrm{RP}_{18}(3.5 \mu \mathrm{m}, 2.1 \mathrm{~mm}$ I.D. $\times 150.0 \mathrm{~mm})$ reversephase analytical column was used with a guard column (Xterra $\mathrm{RP}_{18} 3.5 \mu \mathrm{m}, 2.0 \mathrm{~mm}$ I.D. $\times 10.0 \mathrm{~mm}$ ) (Waters Corporation, Milford MA), and a flow rate of $0.2 \mathrm{~mL} /$ min $(0.3 \mathrm{~mL} / \mathrm{min}$ while washing the $\mathrm{HF}$ residue to the waste). The column pre-filter (Upchurch ultra-low Volume pre-column filter with $0.5 \mu \mathrm{m}$ stainless steel frit, 
Sigma-Aldrich, St. Louis MO) was attached in front of the guard column. Column temperature was $40{ }^{\circ} \mathrm{C}$. The operation and data processing software used was Empower Pro (2002).

The mobile phase was eluted in a gradient mode of $0.88 \%$ formic acid in de-ionized water $(\mathrm{v} / \mathrm{v})(\mathrm{A})$ and methanol (B). In the beginning, 100\% (A) was eluted for $10 \mathrm{~min}$ to the waste from the rear of the guard column. The flow started at $0.2 \mathrm{~mL} / \mathrm{min}$, increased to $0.3 \mathrm{~mL} / \mathrm{min}$ for 2-9 $\mathrm{min}$, and returned to $0.2 \mathrm{~mL} / \mathrm{min}$ at $10 \mathrm{~min}$. Then the mobile phase was switched to elute to the analytical column from the guard column using a switching valve. The gradient system after the switching was as follows: $100 \%$ (A) to $90 \%$ (A) in $8 \mathrm{~min}$ in a linear slope, to $60 \%$ (A) in $7 \mathrm{~min}$ in a linear slope, to $5 \%(\mathrm{~A})$ in $20 \mathrm{~min}$ in a linear slope, to $90 \%$ (A) in $1 \mathrm{~min}$ in a linear slope and held at $90 \%$ (A) for $24 \mathrm{~min}$, and then the flow was switched to the waste, brought to $100 \%$ (A) in 1 min and held at $100 \%$ (A) for $5 \mathrm{~min}$.

\section{Results and discussion Irises}

Over a ground preparation composed principally of lead white, Van Gogh built up the flowers with cobalt blue, zinc white, and eosin red-containing paints, and with Prussian blue and eosin red in the outlines, as reflected in the $\mathrm{Pb}, \mathrm{Co}, \mathrm{Zn}$, and $\mathrm{Br}$ distribution maps presented in Fig. $1 \mathrm{~b}-\mathrm{e}$, and in the Fe distribution map shown in the Additional file 1: Figure S1. The identification of these pigments was corroborated by Raman spectroscopy and SEM-EDS analysis on paint cross-sections, some of which are shown below, and on sample scrapings. Guided by the XRF distribution maps, a limited number of samples for analysis by Raman spectroscopy, SEM-EDS, SERS, and/or HPLC were removed from this painting. The locations of the samples relevant to this article are indicated in Fig. 1a.

Examination of the paint cross-section removed from a flower revealed that the blue and red lake-containing paints were applied wet on wet (Fig. 2a, b). Normal Raman spectra acquired in the red lake pigment layers in this cross-section present bands at ca. 642, 712, 1174, $1280,1345,1501$ and $1620 \mathrm{~cm}^{-1}$, that are consistent with the presence of an eosin lake [25], and a strong feature at $978 \mathrm{~cm}^{-1}$ due to $\mathrm{PbSO}_{4}$ [26]. In Fig. 3a, the spectrum recorded in the red paint layers in this sample (spectrum 1 ) is compared to a spectrum acquired in an eosin red lake reference sample, with barite as a substrate, from a 1900 catalogue of early synthetic dyes [27] (spectrum 2). SEMEDS analysis in the red layers in this sample confirmed the presence of lead sulfate and showed $\mathrm{Br}$ due to eosin red. In the red layer at the bottom of the stratigraphy of the cross-section, $\mathrm{Ca}$ and $\mathrm{Ba}$, most likely originating in calcite and barium white, were detected by SEM-EDS. An SEM image of this sample is presented in Fig. 2c.

Geldof et al. in their study on the use of eosin red lakes in 34 paintings by Van Gogh reported that in all cases in which the inorganic substrate of the lake had been analyzed, Al has been identified [14]. In two of these paintings, Garden with Butterflies and Portrait of Dr. Gachet, both dating to 1890 , these authors identified $\mathrm{Pb}$ and $\mathrm{S}$ most likely in the form of $\mathrm{PbSO}_{4}$ in the eosin lakecontaining paint. They proposed that the compound is formed as a product of the reaction of the dye and aluminum sulfate in the presence of lead acetate, which accords with a 19th century recipe for making the lake, but that it is also possible that $\mathrm{PbSO}_{4}$ was added as an extender.

In this sample, SEM-EDS analysis confirmed the presence of $\mathrm{Co}$ and showed that $\mathrm{Zn}$ is also present in the blue paint. When the cross-section is examined under UV illumination, relatively large pigment particles that fluoresce bright yellow are visible in the blue paint layers (Fig. 2b); in normal illumination they appear an indistinct brownish color. SEM-EDS analysis of these particles showed that they are mainly organic, and that they contain mainly $\mathrm{Zn}$ and $\mathrm{Br}$, in addition to $\mathrm{C}$ (the corresponding spectrum is shown in the Additional file 1: Figure S2. Since all the sample cross-sections were ionmilled, smearing from other paint layers is ruled out as the source of these elements. The normal Raman spectrum acquired in the particles that fluoresce yellow suggests the presence of a lake pigment (Fig. 3a, spectrum 3 ), but this lake could not be firmly identified. The presence of $\mathrm{Br}$ in our sample indicates that the lake might be a compound related to eosin red. Eosin equivalents, with similar absorption spectra but different retention times from eosin and probably formed during the production of the dye, and eosin related components with different absorption spectra than eosin that are either degradation or side products, have been reported in paintings by Van Gogh based on HPLC analyses [14]. According to F.H. Jennison's 1900 catalogue on the manufacture of lake pigments, $\mathrm{ZnSO}_{4} \cdot 7 \mathrm{H}_{2} \mathrm{O}$ was used to precipitate eosin lake pigments, 'but not often' [27]; however, no $\mathrm{S}$ was identified in our sample by SEM-EDS. Other possible sources of $\mathrm{Zn}$ are $\mathrm{ZnO}$, which has also been reported as a substrate in lakes [28], and zinc chloride, that was used as a condensing agent in the manufacture of fluorescein, a starting material for the synthesis of eosin [29]. To our knowledge, $\mathrm{Zn}$ has not been previously identified in substrates of eosin lakes used by Van Gogh, as mentioned above, but, in some cases, this may be explained by the fact that Van Gogh frequently used eosin lake pigments in mixtures with zinc white [14], and that ion milling of sample cross-sections is necessary to rule out its 

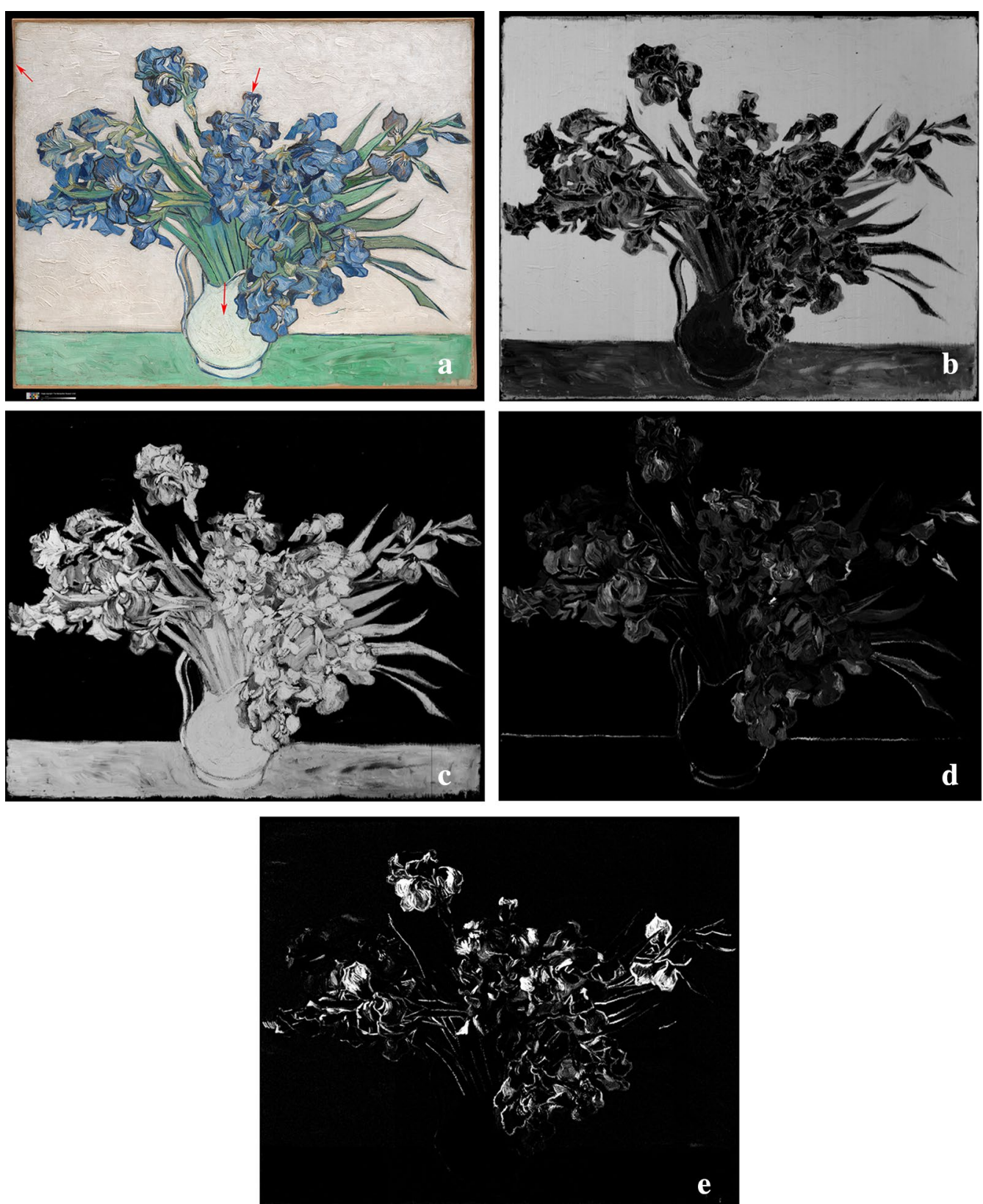

Fig. 1 Irises, 1890 (MMA\#58.187), Vincent van Gogh. Gift of Adele R. Levy, 1958. The arrows show the approximate locations where microsamples for cross-section analysis were removed from $(\mathbf{a}) ; \mathrm{Pb}(\mathbf{b}), \mathrm{Zn}(\mathbf{c}), \mathrm{Co}(\mathbf{d})$, and $\mathrm{Br}(\mathbf{e})$ distribution maps acquired by XRF imaging

interference. To identify the lake pigment, or mixture, in this sample with more certainty, a larger sample would need to be removed for HPLC analysis. If in fact the yellow fluorescent particles contain a red lake, the paint used in the cross-section shown in Fig. 2a, would have been purple.

In the background of the painting, some faint pink strokes are visible with the naked eye, but no clear indication of the presence of $\mathrm{Br}$ was obtained by XRF mapping
(Fig. 1e). Raman spectroscopy and SEM-EDS analyses of a cross-section removed from the left edge of the painting (Fig. 2d-f) showed the wet on wet application of eosin red and lead white paints, that the eosin red lake was precipitated on an $\mathrm{Al}$-containing substrate, and that the small dark pink particles present in this sample contain eosin red on a $\mathrm{CaCO}_{3}$ substrate. It is well known that the inorganic substrates in lake pigments determine in part the stability of the colorants towards the exposure to 


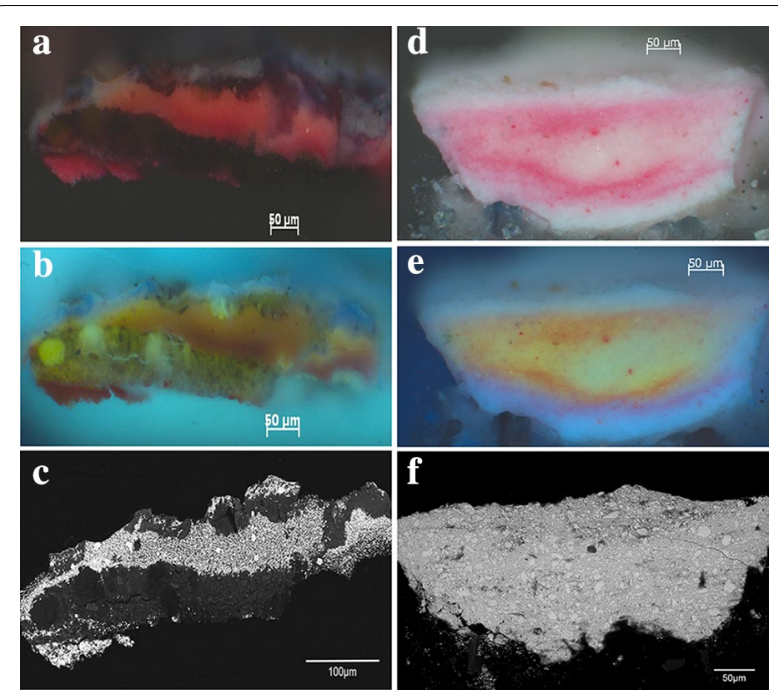

Fig. 2 Irises. Photomicrographs of the paint-cross sections removed from a flower taken with visible (a) and with UV illumination (b), original magnification $\times 200$; and from the discolored background of the painting taken with visible (d) and with UV- illumination (e), original magnification $\times 200$. $\mathbf{c}, \mathbf{f}$ Electron backscattered (BSE) images of the samples shown in $\mathbf{a}$ and $\mathbf{d}$, respectively, taken with $\times 230$ and $\times 245$ magnifications, respectively

light $[11,13]$. To date, no inorganic substrates other than Al-based have been reported for eosin paints in works by Van Gogh [14], and no studies have been published on the permanence of eosin red as precipitated on $\mathrm{CaCO}_{3}$.

SERS (Fig. 3b) and HPLC measurements (Additional file 1: Figure S3) on a microsample removed from the edge of the background confirmed that the only lake pigment used by Van Gogh in this passage is eosin red. SEM-EDS analyses showed that the white 'layer' on top of the stratigraphy is relatively more porous than the lower portion of the sample (Fig. 2f) and that Br- and Alcontaining particles are present together with lead white. These results indicate that this is not a separate layer but part of the pink layer beneath and that it presently looks white due to fading of the eosin red lake.

Van Gogh made multiple orders for eosin paint under the commercial name of laque geranium, or geranium lake, as soon as he arrived in Arles and continued to use it until the end of his life [15].

The possible fading of a red mixed with the light green paint in the vase was also investigated by SEM-EDS and Raman analysis of a paint cross-section and it was found that it was intended to be light green, as it is painted mainly with zinc white with some emerald green and lead white, and no lake pigment particles are present.

Color-calibrated microscopy was used to measure $\mathrm{L} \mathrm{a}^{*} \mathrm{~b} *$ values in the layers composed of eosin red lake in the cross-sections removed from the flower and from

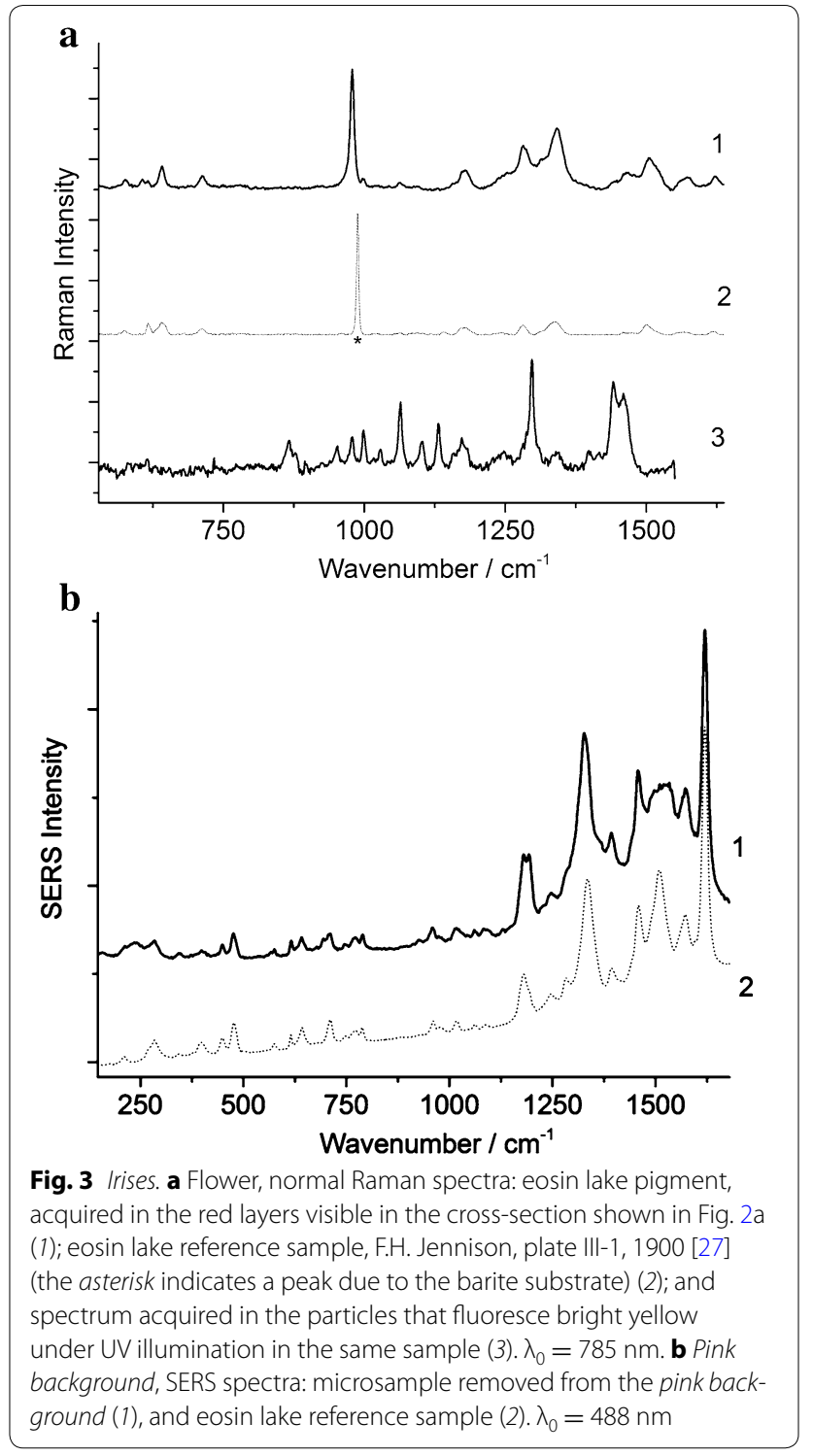

the background of the painting (Fig. 2a, d, respectively). $L * a * b *$ values were also obtained from reflectance spectra acquired in lake paint reconstructions of similar composition prepared by van den Berg et al. following a ninenteenth century recipe $[11,30]$. These color coordinates and the ones obtained by reflectance spectroscopy in the flowers and in areas of the edge that had been protected by an aged brown paper were used for the virtual re-colorization of the painting [1]. Reference was also made to the $\mathrm{X}$-radiograph of the painting and to the $\mathrm{Pb}$ and $\mathrm{Zn}$ distribution maps for the color simulations.

\section{Roses}

Color reproductions of Roses dating from 1928 to 1957 $[1,31]$ were useful for evaluating the relative values 
within the painting but only as subsidiary information to XRF mapping and other imaging techniques for assessing the distribution of the red lakes. The $\mathrm{Br}$ distribution map matches the location of the darkest reds in the flowers but the lightest pink passages did not register (Fig. 4a, c). The pink in the blooms were achieved mainly by applying eosin red and zinc white paints wet on wet and also by mixing these two paints. This complex layering and mixing interfered with and complicated the evaluation of the distribution of the eosin red lake by XRF mapping alone, but the chemical analysis of samples helped to clarify some of these issues and also to evaluate the distribution the brazilwood lake in the tabletop and in the stems.

Results of RIS analysis are presented in Fig. 5 for a detail area in Roses, similar to the detail area shown in Fig. 4b-e. In this area, three red spectral 'endmembers' were found that well describe the dull red observed in the painting. The red paints mapped to portions of the 'white' roses and the roses' buds (Fig. 5a). The red spectral endmembers in the 400 to $580 \mathrm{~nm}$ spectral range show an absorption from $\sim 400$ to $550 \mathrm{~nm}$ with decreasing absorption at longer wavelengths (Fig. 5b). At wavelengths longer than $\sim 580 \mathrm{~nm}$, the red layer becomes increasingly transparent and the underlying paint dominates the reflectance spectra. Two of the red paint endmembers are on a white paint layer and one (bottom spectrum in Fig. $5 \mathrm{~b}$ ) is on a green paint layer of the bud which results in the absorption at wavelengths beyond $\sim 580 \mathrm{~nm}$. In short, all three spectra suggest that a similar red pigment was used in these areas. These spectra have shapes indicative of a red lake pigment [20] rather than a semiconductor, which would have a sharp transition (i.e. vermilion, cadmium reds or red lead) and they do not match iron oxide-based pigments such as Mars red [20]. The combined map shown in Fig. 5a overlays with the deep red and light reds in the color image and, interestingly, with the $\mathrm{Br}$ distribution map in many areas. This also suggests the red pigment used is an eosin-based red lake. Also, the map of the red pigment/s aligns quite well with the strong red features seen in the book-plate image detail in Fig. 4b.

The RIS maps give information in the visible at or near the paint surface as the optimal depth depends on the absorption and scattering properties of the outer paint
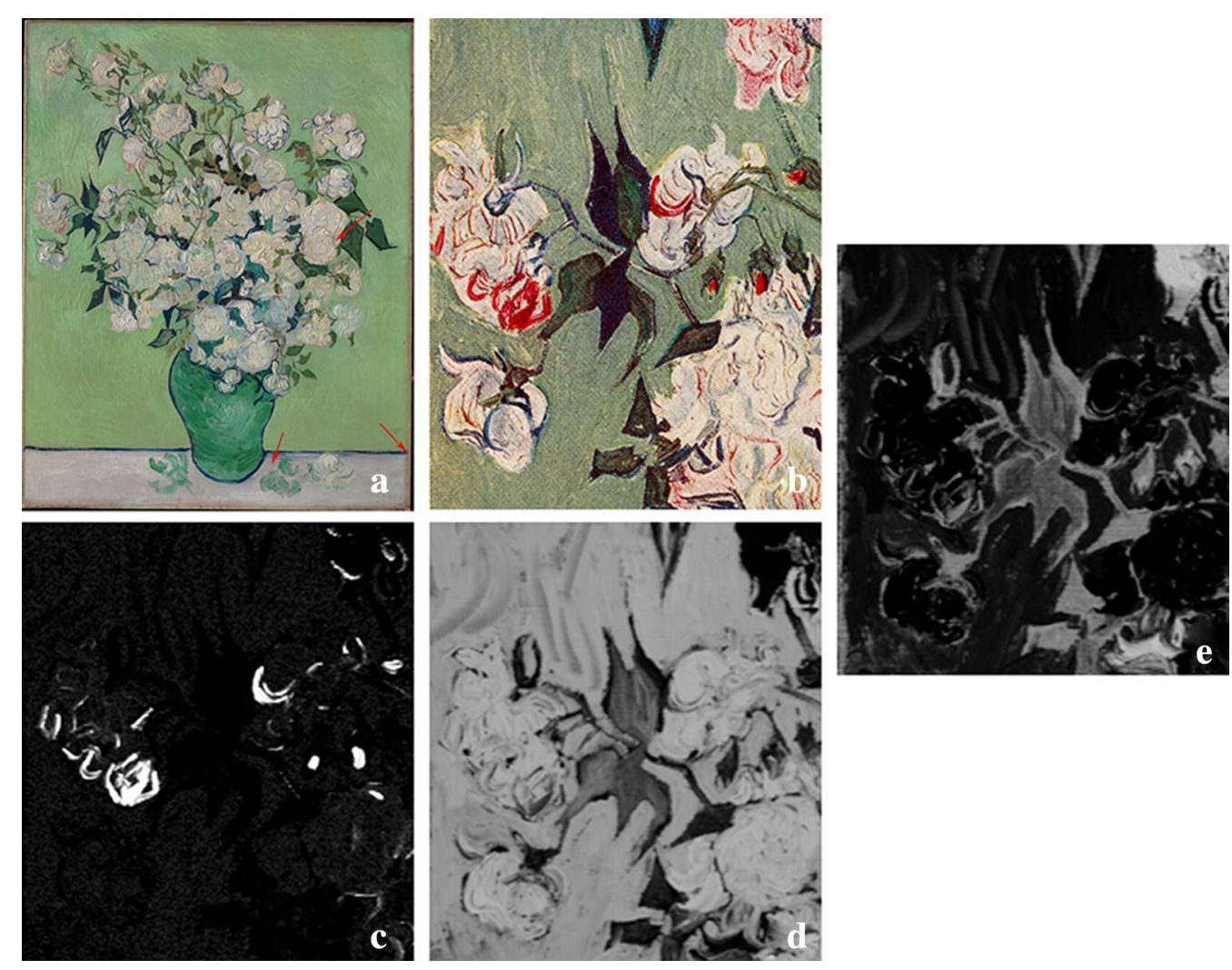

Fig. 4 Roses, 1890 (MMA\#1993.400.5), Vincent van Gogh. The Walter H. and Leonore Annenberg Collection, Gift of Walter H. and Leonore Annenberg, 1993, Bequest of Walter H. Annenberg, 2002. The arrows show the locations where samples for cross-section analysis were removed (a). Details of book plate from Recueil important des oeuvres de Vincent Van Gogh, Tokio, Atelier, 1935 [5] (b); and of Br (c), Zn (d) and Pb (e) distribution maps acquired by XRF imaging. The image $\mathbf{b}$ is cropped on the left edge in the original publication 

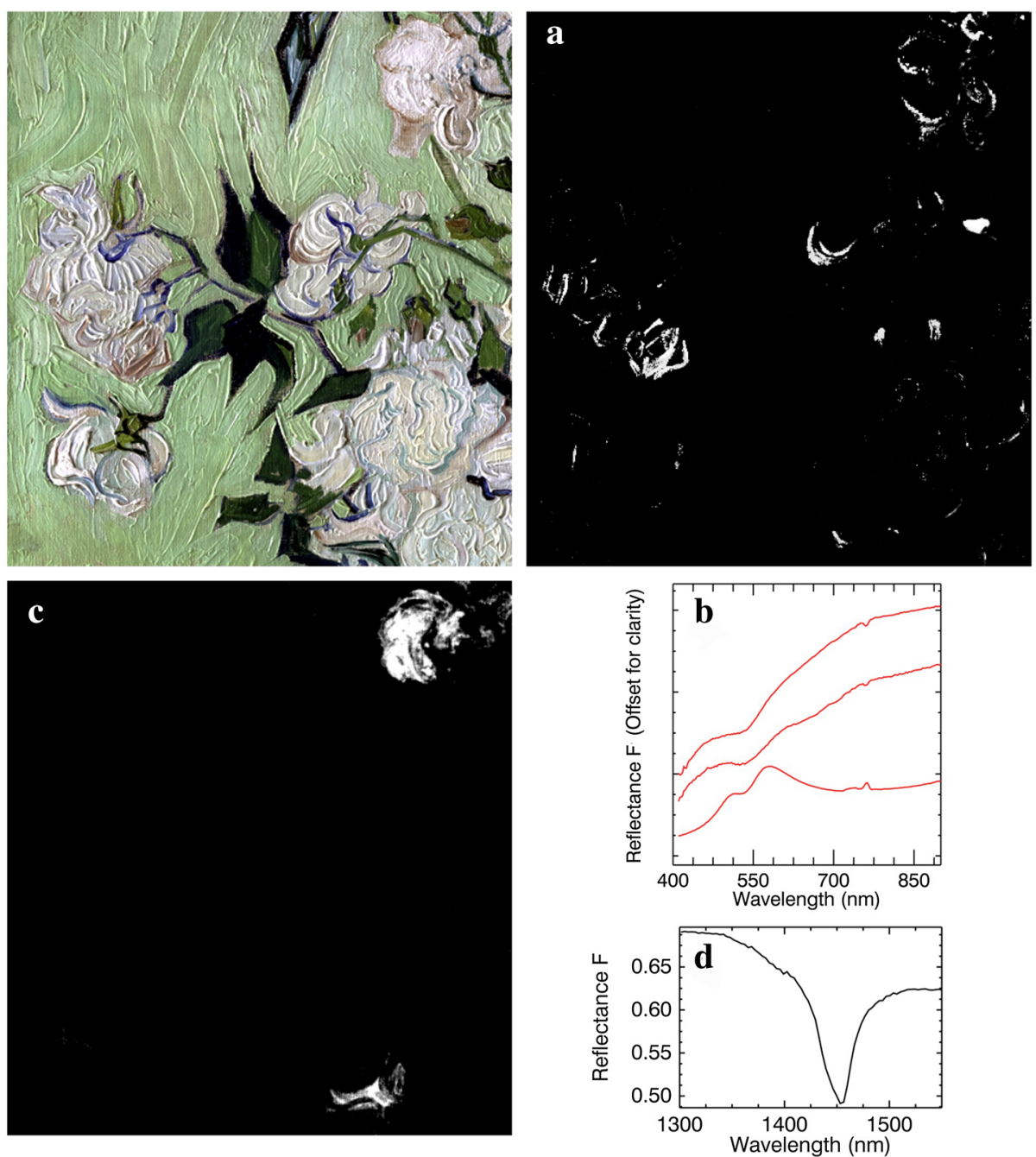

Fig. 5 Roses. Reflectance imaging spectroscopy (RIS) analysis of the detail area shown at top left. Map (a) and diffuse reflectance spectra (b) of red end members. Map (c) and diffuse reflectance spectrum (d) of lead white

layer whereas the XRF distribution maps can provide information at layers below the surface paints (noting that the matrix effect modulates this). This likely explains the few differences observed between the Br distribution map obtained by XRF mapping and the RIS maps for the reds. This is apparent in the comparison of the XRF distribution map for $\mathrm{Pb}$ and the RIS map due to lead white (Figs. 4e, 5c).

For mapping the presence of lead white (basic lead carbonate) the narrow feature at $1446 \mathrm{~nm}$ (Fig. 5d), which originates from the hydroxyl first overtone, was used [32]. The lead white used by Van Gogh is most likely a commercial product, expected to have a larger proportion of the basic lead carbonate over lead carbonate. In the RIS map, the $1446 \mathrm{~nm}$ feature is localized to one flower (top right) and a portion of the lower right flower
(Fig. 5c). The white pigment for the other flowers is likely zinc white given the XRF distribution map for $\mathrm{Zn}$ (Fig. 4d) and the finding of strong absorption from 350 to $\sim 370 \mathrm{~nm}$ in the site specific fiber optics reflectance spectra.

SERS and HPLC analyses of a microsample removed from the edge of the pink tabletop that had been protected from light exposure by the frame rebate (Fig. 4a) showed that Van Gogh used brazilwood, and that no cochineal or any other organic colorant is present. Brazilwood was also identified by SERS and HPLC in a microsample removed for the dark red paint used for some of the stems, in which the lake-used without the admixture of white-does not appear to have faded to the extent observed in the flowers. The corresponding SERS spectra are shown in Fig. 6 together with the spectrum of 
a brazilwood reference, and the HPLC data is included in Additional file 1: Figure S4. Brazilwood and eosin lakes are the most fugitive red lake pigments among others used by Van Gogh such as cochineal lake [11]. Therefore, carminic acid, the main component of cochineal and a more stable colorant, would have been detected by HPLC analysis if it was present in the sample. Burnstock et al. have reported that the most severe fading tends to occurs where the lakes are mixed with lead white or zinc white [11], as observed in the blooms.

Geldof et al. in their study of Van Gogh's palette in the last period of his life (1888-1890) found that, starting in the summer of 1889, Van Gogh used a paint containing a redwood colorant and cochineal precipitated on an $\mathrm{Al} /$ Sn-containing substrate, with a small amount of $\mathrm{CaCO}_{3}$, and state that he likely bought this as a ready-made paint mixture since they did not identify redwood separately [15]. Redwood alone had been previously identified in Garden of the Asylum [12] by thin layer chromatography; both lakes were identified separately in paintings done while Van Gogh was in Paris [13].

Two microsamples were removed from the tabletop and mounted as cross-sections for Raman and SEM-EDS measurements. The first sample was taken from an area where the pink paint is protected by the blue horizon line, on the right edge of the painting, and the second one from a spot where the red lake pigment has visibly faded (Fig. 4a). In the first sample, the pink layer is applied over the white ground preparation containing lead white and $\mathrm{CaCO}_{3}$ (Fig. 7a, b). Raman analysis of the pink layer showed that the brazilwood lake is mixed with zinc white, while SEM-EDS revealed that the lake is precipitated onto a substrate composed of $\mathrm{Sn}$ and $\mathrm{Al}$, and that there are a few $\mathrm{CaCO}_{3}$ particles in this layer. It should be

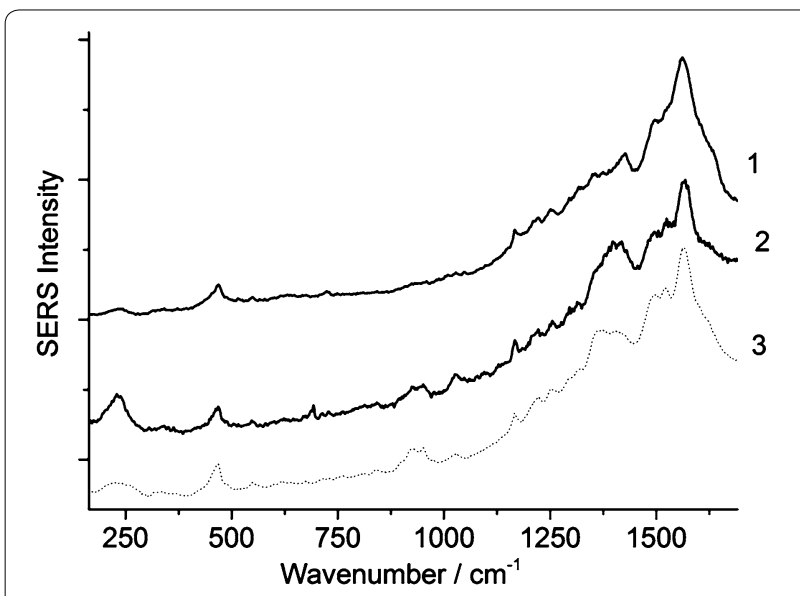

Fig. 6 Roses. SERS spectra of red lake pigment in the tabletop (1), stem (2), and brazilwood lake reference (3). $\lambda_{0}=488 \mathrm{~nm}$ noted that, in the pink layer, the red colorant has faded in the spot where the Prussian blue paint layer on top of the stratigraphy has a gap; this spot is indicated by an arrow in the photomicrograph presented in Fig. 7a.

The second sample cross-section removed from the tabletop is missing the ground preparation layer (Fig. 7c, d). In the dark pink layer at the bottom of the stratigraphy, the brazilwood lake is precipitated onto a substrate containing $\mathrm{Sn}$ and $\mathrm{Al}$ and it is mixed with zinc white and few $\mathrm{CaSO}_{4}$ particles. In the white layer at the top of the sample, relatively bigger $\mathrm{CaSO}_{4}$ particles, $\mathrm{CaCO}_{3}$ particles, relatively smaller amounts of particles containing $\mathrm{Sn}$ and $\mathrm{Al}$, also smaller in size, and larger amounts of zinc white were detected by SEM-EDS when compared to the dark pink layer beneath. The presence of particles containing $\mathrm{Sn}$ and $\mathrm{Al}$ in this white layer indicates that it must have been colored by a brazilwood lake similar to the one in the darker pink layer beneath and that this colorant has now faded. The relatively smaller amount of particles containing $\mathrm{Sn}$ and $\mathrm{Al}$ and the larger zinc white content in this white layer indicates that, originally, it must have been of a paler pink.

In a sample removed from a rose in the right side of the painting (Fig. 4a), eosin red and zinc white-containing paints, applied wet on wet, were identified by Raman spectroscopy and $\mathrm{PbSO}_{4}$ was found to be present in the red paint by Raman and SEM-EDS (Fig. 7e, f). The composition of the red paint in this sample shown from Roses is similar to the one observed in the sample taken from a flower in Irises and shown in Fig. $2 \mathrm{a}-\mathrm{c}$.

For the virtual color simulation of the tabletop, $L^{*} \mathrm{a}^{*} \mathrm{~b}$ values were obtained by calibrated microscopy in the cross-section shown Fig. 7a, where the eosin red lake is protected by the Prussian blue layer, and by calibrated photography in the right edge of the painting, in an area that had been protected from exposure to light. For the color simulations of the flowers, the $L^{*} a^{*} b^{*}$ values used were those measured in the cross-section shown in Fig. 7e and those obtained in multiple spots in the roses by reflectance spectroscopy [1]. Reference was also made to the X-radiograph of the painting and to the $\mathrm{Pb}$ and $\mathrm{Zn}$ distribution maps for the color simulations.

\section{Conclusions}

Imaging and microchemical analysis of Van Gogh's still lifes, Irises and Roses, not only helped further understanding of Van Gogh's working practice but also revealed to what extent different paint passages containing fugitive red lake pigments had changed. Fading of the red lakes has resulted in pink paints that now look white and in purple paints that now look blue. Since the works are part of an ensemble, the changes in each individual painting also affect the way we perceive them as a group. 

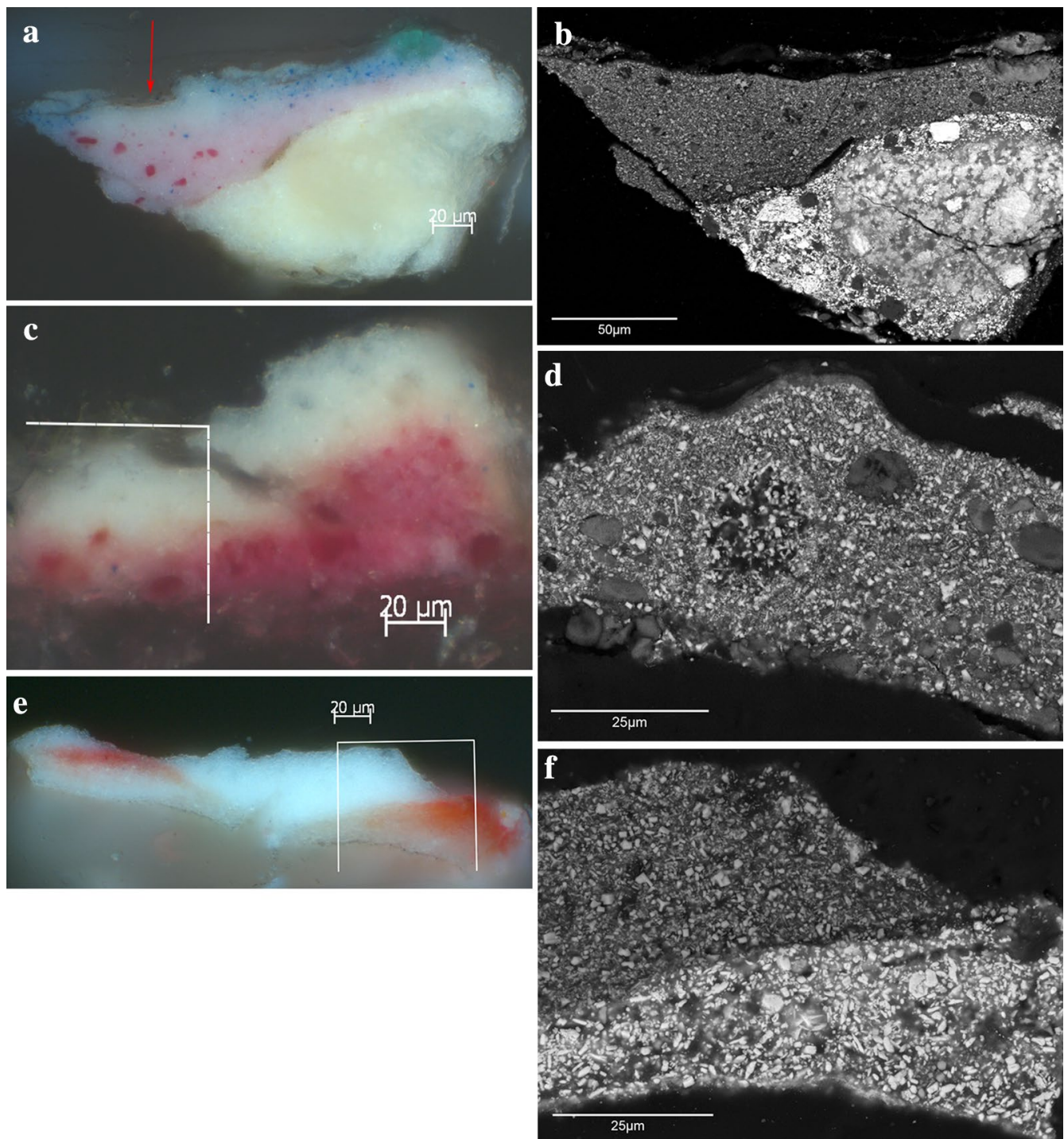

Fig. 7 Roses. Photomicrographs taken with visible illumination of paint cross-sections removed from a protected area in the edge of the tabletop (a); from a visibly faded area in the tabletop (c); and from a flower (e), all taken with a $\times 400$ magnification. In $\mathbf{a}$, the arrow indicates a spot where the blue paint layer at the top of the cross-section has a gap and the pink lake pigment in the layer below has faded. $\mathbf{b}$, $\mathbf{d}$, $\mathbf{f}$ are electron backscattered (BSE) images of the samples shown in $\mathbf{a}, \mathbf{c}, \mathbf{e}$, respectively. Image (b) was taken with $\times 555$ magnification; (d) is a detail taken on the left side of the sample with a $\times 1400$ magnification (the location is indicated in $\mathbf{c}$ by white lines); and $\mathbf{f}$ is a detail taken on the right side of the sample with a $\times 1420$ magnification (the location is indicated in e by white lines)

Studies like this provide new art historical perspectives and offer modern viewers a sense of how the works originally looked.

\section{Additional file}

Additional file 1. Additional figures.

\section{Authors' contributions}

SC, FC, AC and NS contributed to the acquisition of XRF, Raman, SERS, SEMEDS and HPLC data, processing, and interpretation; $\mathrm{CH}$ contributed to the interpretation of data; JKD and KD carried out the RIS measurements and the interpretation of the data; GvdS and KJ processed XRF mapping data with PyMca and Datamuncher software; SS contributed with art historical perspective. All authors were involved in drafting the manuscript. All authors read and approved the final manuscript.

\section{Author details}

${ }^{1}$ Department of Scientific Research, The Metropolitan Museum of Art, 1000 Fifth Avenue, New York, NY 10028, USA. ${ }^{2}$ Department of Paintings Conservation, The Metropolitan Museum of Art, 1000 Fifth Avenue, New York, NY 10028, USA. ${ }^{3}$ Scientific Research Department, National Gallery of Art, Washington, DC, USA. ${ }^{4}$ AXES Research Group, Department of Chemistry, University of Antwerp, Antwerp, Belgium. ${ }^{5}$ Department of European Paintings, The Metropoli$\tan$ Museum of Art, 1000 Fifth Avenue, New York, NY 10028, USA. 


\section{Acknowledgements}

Numerous colleagues contributed to the different aspects of the technical examination and to the color simulations: Barbara Berrie, Damon Conover, Ann Hoenigswald, and Giorgio Trumpy at the National Gallery of Art, Washington; Ella Hendriks, Teio Meedendorp, and Louis van Tilborgh at the Van Gogh Museum, Amsterdam; Michael Gallagher, Scott Geffert, Chris Heins, Evan Read, and Juan Trujillo at The Met; Aviva Burnstock at the Courtauld Institute of Art, and Klaas Jan van den Berg, at the Cultural Heritage Agency of The Netherlands.

Francesca Casadio, at the Art Institute of Chicago, kindly shared with us a sample of a Kopp's purpurin lake paint reconstruction prepared and provided by Klaas Jan van den Berg, at the Cultural Heritage Agency of The Netherlands. We are indebted to both colleagues for their generosity. This paint reconstruction was prepared as part of a project involving the two institutions mentioned along with the Shell Research and Technology Center, Amsterdam, the Courtauld Institute and the National Gallery, London.

We are also thankful to Joris Dik, Delft University of Technology, and Koen Janssens, University of Antwerp, for the loan of an XRF scanner prototype to The Metropolitan Museum of Art.

\section{Competing interests}

The authors declare that they have no competing interests.

\section{Publisher's Note}

Springer Nature remains neutral with regard to jurisdictional claims in published maps and institutional affiliations.

Received: 19 January 2017 Accepted: 3 April 2017

Published online: 10 May 2017

\section{References}

1. Stein SA, Hale C. Van Gogh, Irises and Roses. 2015. http://www.metmuseum.org/metmedia/video/collections/ep/van-gogh-irises-and-roses.

2. Aceto M, Agostino A, Fenoglio G, Idone A, Gulmini M, Piccolo M, et al. Characterisation of colorants on illuminated manuscripts by portable fibre optic UV-visible-NIR reflectance spectrophotometry. Anal Methods. 2014;6:1488.

3. Clementi C, Doherty B, Gentili PL, Miliani C, Romani A, Brunetti BG, et al. Vibrational and electronic properties of painting lakes. Appl Phys A. 2008;92(1):25-33.

4. Leona M. Microanalysis of organic pigments and glazes in polychrome works of art by surface-enhanced resonance Raman scattering. Proc Natl Acad Sci USA. 2009:106(35):14757.

5. Casadio F, Leona M, Lombardi JR, Van Duyne R. Identification of organic colorants in fibers, paints, and glazes by surface enhanced Raman spectroscopy. Acc Chem Res. 2010;43(6):782-91.

6. Kirby J, White R. The identification of red lake pigment dyestuffs and a discussion of their use. Natl Gall Tech Bull. 1996:17:56-80.

7. Degano I, Ribechini E, Modugno F, Colombini MP. Analytical methods for the characterization of organic dyes in artworks and in historical textiles. Appl Spectrosc Rev. 2009;44:363-410.

8. Wyplosz N. Laser desorption mass spectrometric studies of artists' organic pigments. University of Amsterdam; 2003.

9. Ghelardi E, Degano I, Colombini MP, Mazurek J, Schilling M, Learner T. Py-GC/MS applied to the analysis of synthetic organic pigments: characterization and identification in paint samples. Anal Bioanal Chem. 2015;407(5):1415-31.

10. Vellekoop M, Geldof M, Hendriks E, Jansen L, deTagle A, editors. Van Gogh's studio practice. Brussels Mercatorfonds; 2013.

11. Burnstock A, Lanfear I, van den Berg KJ, Carlyle L, Clarke M, Hendriks E, et al. Comparison of the fading and surface deterioration of red lake pigments in six paintings by Vincent van Gogh with artificially aged paint reconstructions. In: Paterakis AB, editor. ICOM 14th Triennial Meeting The Hague: ICOM Committee for Conservation; 2005. p. 459-66.
12. Hendriks E, Van Tilborgh L. Van Gogh's 'Garden of the Asylum': genuine or fake? Burlingt Mag. 2001;143(1176):145-56.

13. van Bommel $M$, Geldof $M$, Hendriks E. An investigation of organic red pigments used by Vincent van Gogh (November 1885 to February 1888). Art Matters. 2005:3:111-37.

14. Geldof M, de Keijzer M, van Bommel M, Pilz K, Salvant J, van Keulen H, et al. Van Gogh's Geranium Lake. In: Vellekoop M, Geldof M, Hendriks E, Jansen L, deTagle A, editors. Van Gogh's studio practice. Brussels: Mercatorfonds; 2013.

15. Geldof M, Megens L, Salvant J. Van Gogh's Palette in Arles, Saint-Rémy and Auvers-sur-Oise. In: Vellekoop M, Geldof M, Hendriks E, Jansen L, de Tagle A, editors. Van Gogh's studio practice. Brussels: Mercatorfonds; 2013.

16. Groom G, editor. Van Gogh's bedrooms. Chicago: The Art Institute of Chicago; 2016

17. Alfeld M, Janssens K. Strategies for processing mega-pixel X-ray fluorescence hyperspectral data: a case study on a version of Caravaggio's painting Supper at Emmaus. J Anal Atomic Spectrom. 2015;30(3):777-89. doi:10.1039/C4JA00387J.

18. Dooley KA, Conover DM, Glinsman LD, Delaney JK. Complementary standoff chemical imaging to map and identify artist materials in an early Italian Renaissance panel painting. Angew Chem Int Ed. 2014;53(50):13775-9.

19. Conover DM, Delaney JK, Loew MH. Automatic registration and mosaicking of technical images of Old Master paintings. Appl Phys A 2015;119(4):1567-75

20. Delaney JK, Zeibel JG, Thoury M, Littleton R, Palmer M, Morales KM, et al. Visible and infrared imaging spectroscopy of Picasso's Harlequin musician: mapping and identification of artist materials in situ. Appl Spectrosc 2010:64(6):584-94.

21. Boardman JW, Kruse FA, Green RO. Mapping target signatures via partia unmixing of AVIRIS data. Summaries, Fifth JPL Airborne Earth Science Workshop. JPL Publication; 1995. p. 23-6.

22. Cesaratto A, Pozzi F, Leona M. Sample treatment considerations in the analysis of organic colorants by surface-enhanced Raman scattering. Part II: synthetic dyes Unpublished results.

23. Pozzi F, Lombardi JR, Bruni S, Leona M. Sample treatment considerations in the analysis of organic colorants by surface-enhanced Raman scattering. Anal Chem. 2012;84(8):3751-7.

24. Sanyova J. Mild extraction of dyes by hydrofluoric acid in routine analysis of historical paint micro-samples. Microchim Acta. 2008;162(3):361-70.

25. Whitney AV, Van Duyne RP, Casadio F. An innovative surface-enhanced Raman spectroscopy (SERS) method for the identification of six historical red lakes and dyestuffs. J Raman Spectrosc. 2006;37(10):993-1002.

26. Burgio L, Clark RJH. Library of FT-Raman spectra of pigments, minerals, pigment media and varnishes, and supplement to existing library of Raman spectra of pigments with visible excitation. Spectrochim Acta Part A Mol Biomol Spectrosc. 2001;57(7):1491-521.

27. Jennison $\mathrm{FH}$. The manufacture of lake pigments from artificial colours. London: Scott, Greenwood \& Son, New York, Van Nostrand; 1900.

28. Jennison $\mathrm{FH}$. The manufacture of lake pigments from artificial colours. 2nd ed. London: Scott, Greenwood \& Son, New York, Van Nostrand; 1920

29. Baeyer A. Ueber eine neue Klasse von Farbstoffen. Ber Dtsch Chem Ges. 1871:4(2):555-8

30. Van den Berg KJ, Burnstock A, Carlyle L, Clarke M, Hendriks E, Hoppenbrouwers $\mathrm{R}$, et al. Fading of red lake paints after Vincent van Gogh-an interdisciplinary study involving three de Mayerne projects. In: Boon JJ, Ester SB, editors. Reporting highlights of the De Mayerne Programme Research programme on molecular studies in conservation and technical studies in art history. The Hague: Netherlads Organisation for Scientific Research (NWO); 2006

31. Van Gogh V, Hazama I. Recueil important des oeuvres de Vincent Van Gogh: Tokio, Atelier; 1935.

32. Picollo M, Bacci M, Magrini D, Radicati B, Trumpy G, Tsukada M, et al. Modern white pigments: their identification by means of noninvasive ultraviolet, visible, and infrared fiber optic reflectance spectroscopy. Proceedings from the modern paints uncovered symposium; 2007. p. 118-28. 